УДК 538.956

\title{
ДОМЕННЫЙ ВКЛАД В ДИЭЛЕКТРИЧЕСКИЕ СВОЙСТВА КРИСТАЛЛОВ ГРУППЫ ТРИГЛИЦИНСУЛЬФАТА И СЕГНЕТОВОЙ СОЛИ
}

\author{
(C) 2017 О. М. Голицына, С. Н. Дрождин, В. О. Чулакова, А. Е. Гриднев \\ Воронежский государственный университет, Университетская пл., 1, 394018 Воронеж, Россия \\ e-mail:drozhdin@phys.vsu.ru
}

Поступила в редакцию 31.03 .2017 г.

\begin{abstract}
Аннотация. Исследованы диэлектрические свойства кристаллов номинально чистого ТГС, дейтерированного ТГС, ТГС с примесью $L, \alpha$ - аланина, ТГС с примесью ионов фосфора $\mathrm{P}^{5+}$, ТГС, подвергнутого рентгеновскому облучению; кристалла сегнетовой соли. Получены температурные зависимости доменных компонент действительной и мнимой частей диэлектрической проницаемости, измеренных в слабом переменном электрическом поле. Существование в бездефектных кристаллах группы ТГС подвижной доменной структуры не приводит к отклонению от термодинамического закона Кюри-Вейсса в сегнетоэлектрической фазе, но вызывает нарушение «закона двойки». В кристаллах ТГС с примесными или радиационными дефектами, закрепляющими доменную структуру, хорошо выполняются оба закона. Для исследованных кристаллов группы ТГС вблизи точки фазового перехода отсутствует количественное совпадение между температурным поведением статических геометрических параметров доменной структуры (плотность $N$ и полная длина $L$ доменных границ) и измеряемых диэлектрических характеристик. Число термических циклов нагревания - охлаждения с переходом через точку Кюри практически не влияет на величину доменной составляющей диэлектрической проницаемости в кристаллах ТГС, а увеличение скорости прохождения фазового перехода приводит к ее заметному понижению. В кристалле сегнетовой соли доменная часть диэлектрической проницаемости вблизи нижнего фазового перехода заметно меньше чем вблизи верхнего, но занимает более широкий температурный интервал.
\end{abstract}

Ключевые слова: триглицинсульфат, сегнетова соль, доменная структура, осцилляции доменных границ, диэлектрические свойства, дефекты, закон Кюри-Вейсса, «закон двойки».

\section{ВВЕДЕНИЕ}

Уникальные физические свойства и соответствующие макроскопические характеристики сегнетоэлектриков в значительной мере определяются поведением доменной структуры при изменении внешних параметров - электрического поля, механических напряжений, температуры. В частности, значения диэлектрических характеристик существенно зависят от вклада осцилляций доменных границ, а именно, от многочисленных актов локальной пристеночной переполяризации, в измеряемый отклик кристалла на действие переменного электрического поля [1]. Характер доменной структуры, возникшей при фазовом переходе, и ее дальнейшее поведение определяются целым рядом факторов, в частности, дефектностью кристаллической решетки и особенностями перевода сегнетоэлектрика через точку Кюри $T_{\mathrm{C}}[1,2]$. С одной стороны, представляет интерес выявление величины и роли доменной компоненты измеряемых макроскопических параметров, а с другой, изучение по их полевым, температурным, временным и иным зависимостям особенностей поведения самой доменной структуры с учетом указанных факторов.

Наиболее ярко значительный доменный вклад в низкочастотные диэлектрические свойства проявляется в сегнетоэлектрических кристаллах группы дигидрофосфата калия, для которых этот феномен всесторонне исследован экспериментально [3] и обоснован теоретически [1]. Для других хорошо изученных модельных сегнетоэлектриков, таких как триглицинсульфат (ТГС), сегнетова соль (CC), титанат бария, ниобат лития и др., существует значительно меньше экспериментальной информации. 
Целью настоящей работы явилось исследование особенностей проявления доменной компоненты низкочастотного диэлектрического отклика кристаллов триглицинсульфата - номинально чистого и с дефектами разной природы, а также кристалла сегнетовой соли.

\section{ОБЪЕКТЫ И МЕТОДИКИ ИССЛЕДОВАНИЯ}

Исследовались кристаллы номинально чистого ТГС; дейтерированного ТГС с содержанием дейтерия в растворе $\sim 80 \%$ - ДТГС; ТГС с примесью $L, \alpha$-аланина $(10 \% L, \alpha$-аланина в растворе $)-$ АТГС; ТГС с примесью ионов фосфора $\mathrm{P}^{5+}$ (весовое содержание триглицинфосфата $\left(\mathrm{NH}_{2} \mathrm{CH}_{2} \mathrm{COOH}_{3}\right) \cdot \mathrm{H}_{3} \mathrm{PO}_{4}$ в растворе $\sim 50 \%$ ) - ТГСФ; ТГС, подвергнутого рентгеновскому облучению - XR-TГC; кристалла сегнетовой соли. Рентгеновское облучение $(\mathrm{CuK}$, энергия 30 кэВ, экспозиционная мощность дозы $N_{\mathrm{D}}=240 \mathrm{kR} \cdot \mathrm{h}^{-1}$ ) проводилось поэтапно с постепенным накоплением дозы с шагом $24 \mathrm{kR}$. Измерения проводились спустя $\sim 180$ часов после облучения, необходимых для стабилизации системы созданных радиационных дефектов [4]. Спустя 240 часов после проведенных измерений образцы облучались повторно и измерения проводились так же, как и после первого облучения. Максимальная доза облучения составляла $240 \mathrm{kR}$.

Монокристалл ТГС (химическая формула $\left.\left(\mathrm{NH}_{2} \mathrm{CH}_{2} \mathrm{COOH}_{3}\right) \cdot \mathrm{H}_{2} \mathrm{SO}_{4}\right)$ испытывает сегнетоэлектрический фазовый переход второго рода $\mathrm{P} 2 / \mathrm{m} \rightarrow \mathrm{P} 2_{1}$ при $T_{C}=49^{\circ} \mathrm{C}$ с возникновением спонтанной поляризации $\boldsymbol{P}_{S}$ вдоль полярной оси $\boldsymbol{b}$ и появлением $180^{\circ}$-ной доменной структуры. В кристалле ДТГС- $\left(\mathrm{ND}_{2} \mathrm{CD}_{2} \mathrm{COOD}_{3}\right) \cdot \mathrm{D}_{2} \mathrm{SO}_{4}$ температура сегнетоэлектрического фазового перехода второго рода $\mathrm{P} 2 / 1 \mathrm{~m} \rightarrow \mathrm{P} 2_{1}$ зависит от степени дейтерирования и в нашем случае равнялась $57.5^{\circ} \mathrm{C}$. В кристаллах АТГС и ТГСФ температуры фазового перехода, определенные по положению максимума температурной зависимости диэлектрической проницаемости, составляли, соответственно 49.3 и $48.7^{\circ} \mathrm{C}$.

Сегнетова соль $\left(\mathrm{KNaC}_{4} \mathrm{H}_{4} \mathrm{O}_{6} \cdot 4 \mathrm{H}_{2} \mathrm{O}\right)$ - монокристалл с двумя фазовыми переходами второго рода $\mathrm{P} 2{ }_{1} 2{ }_{1} \rightarrow \mathrm{P} 2{ }_{1}$ при температурах $T_{\mathrm{Cl}}=-18{ }^{\circ} \mathrm{C}$ и $T_{\mathrm{C} 2}=+24{ }^{\circ} \mathrm{C}$. Спонтанная поляризация возникает вдоль полярной оси $\boldsymbol{a}$, совпадающей с винтовой осью второго порядка, лежащей вдоль кристаллографического направления [100]. В кристаллах СС сосуществуют домены двух типов: $a b$ - и $a c$-домены, границы которых расположены, соответствен- но, параллельно ромбическим плоскостям (001) и (010), причем $a b$-домены более стабильны по отношению к внешним воздействиям.

Образцы для исследований (срезы (010) кристаллов группы ТГС и (100) - кристалла СС) со средними размерами $0.5 \times 0.5 \times 0.1 \mathrm{~cm}^{3}$ длительно выдерживались в разомкнутом состоянии при комнатной температуре, а затем на них в вакууме были напылены серебряные электроды.

Электрическая емкость, проводимость и тангенс угла диэлектрических потерь измерялись в слабом переменном электрическом поле (амплитуда $3 \mathrm{~B} \cdot \mathrm{cm}^{-1}$, частота 1000 Гц) с помощью измерителя импеданса Wayne Kerr WK 4270. Погрешность измерения этих величин не превышала $5 \%$. По измеренным параметрам рассчитывались действительная $\varepsilon^{\prime}$ и мнимая $\varepsilon^{\prime \prime}$ части диэлектрической проницаемости.

Образцы исследуемых кристаллов «омолаживались» кратковременной выдержкой в параэлектрической фазе. Для этого образцы кристаллов группы ТГС начиная с $T=40^{\circ} \mathrm{C}$ медленно нагревались со скоростью $\sim 0.1 \mathrm{~K} \cdot$ мин $^{-1}$, переводились в парафазу, выдерживались 30 мин при $T=60{ }^{\circ} \mathrm{C}$ и затем охлаждались с той же скоростью в сегнетоэлектрическую фазу до заданной температуры. Образцы кристалла СС со скоростью $\sim 0.1 \mathrm{~K}$ мин $^{-1}$ переводились в неполярные фазы до температур, соответственно +30 и $-24{ }^{\circ} \mathrm{C}$, выдерживались там примерно 30 мин и затем медленно возвращались в сегнетоэлектрическую фазу до заданной температуры.

\section{ЭКСПЕРИМЕНТАЛЬНЫЕ РЕЗУЛЬТАТЫ И ИХ ОБСУЖДЕНИЕ}

\section{1. Кристаллы группы ТГС}

Диэлектрические параметры полидоменного сегнетоэлектрика, измеряемые в переменном электрическом поле, являются суммой решеточной и доменной компонент, например, диэлектрическая проницаемость $\varepsilon_{\text {изм }}(T)=\varepsilon_{\text {реш }}(T)+\varepsilon_{\text {дом }}(T)$ [5-8]. Доменная компонента присутствует всегда в сегнетоэлектрической фазе, где решеточная составляющая неизвестна, не может быть заменена ее величиной, измеренной в параэлектрической фазе, где $\varepsilon_{\text {изм }}(T)=\varepsilon_{\text {реш }}(T)$, и поэтому возникает проблема экспериментального выделения $\varepsilon_{\text {дом }}(T)$. Температурной зависимостью величины, в наибольшей степени соответствующей доменной компоненте параметра, может служить разность двух его температурных зависимостей: измеренной при охлаждении в сегнетоэлектричес- 
кую фазу образца, выдержанного в параэлектрической фазе, и измеренной при нагревании образца, выдержанного в сегнетоэлектрической фазе вдали от $T_{\mathrm{C}}: \varepsilon_{\text {изм }}(T)_{\text {охл }}-\varepsilon_{\text {изм }}(T)_{\text {нагр }}=\varepsilon_{\text {реш }}(T)_{\text {охл }}-\varepsilon_{\text {реш }}(T)_{\text {нагр }}+$ $+\varepsilon_{\text {дом }}(T)_{\text {охл }}-\varepsilon_{\text {дом }}(T)_{\text {нагр }}[5,6]$. Можно считать, что температурные зависимости решеточной части при нагревании и охлаждении одинаковы, поэтому $\varepsilon_{\text {дом }}(T) \approx \Delta \varepsilon_{\text {изм }}(T)=\varepsilon_{\text {дом }}(T)_{\text {охл }}-\varepsilon_{\text {дом }}(T)_{\text {нагр }}$. Для полидо-

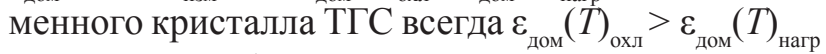
поскольку в образце, длительно выдержанном в сегнетоэлектрической фазе, доменная структура стабильна, доменные стенки закреплены естественными дефектами, малоподвижны, а их общая длина невелика. В этом же образце, «омоложенном» переводом в параэлектрическую фазу, при последующем охлаждении в полярную фазу возникает неравновесная мелкая доменная структура с большим числом и большой общей длиной доменных стенок. Естественно, что вклад такой доменной структуры в величину измеряемых параметров будет существенно выше. Разность $\Delta \varepsilon_{\text {изм }}(T)$ будет в наибольшей степени соответствовать $\varepsilon_{\text {дом }}(T)$, если будет минимальна $\varepsilon_{\text {дом }}(T)_{\text {нагр }}$ и максимальна $\varepsilon_{\text {дом }}(T)_{\text {охл }}$. Доменную компоненту, в принципе, можно уменьшить (сделать равной нулю), монодоменизируя кристалл постоянным электрическим полем, последующее выключение которого стимулирует ярко выраженный релаксационный процесс деполяризации с соответствующим изменением диэлектрических параметров $[9,10]$, т.е. существенную роль в исследованиях будет играть еще один фактор - время. Этот фактор присутствует и в обычных измерени-

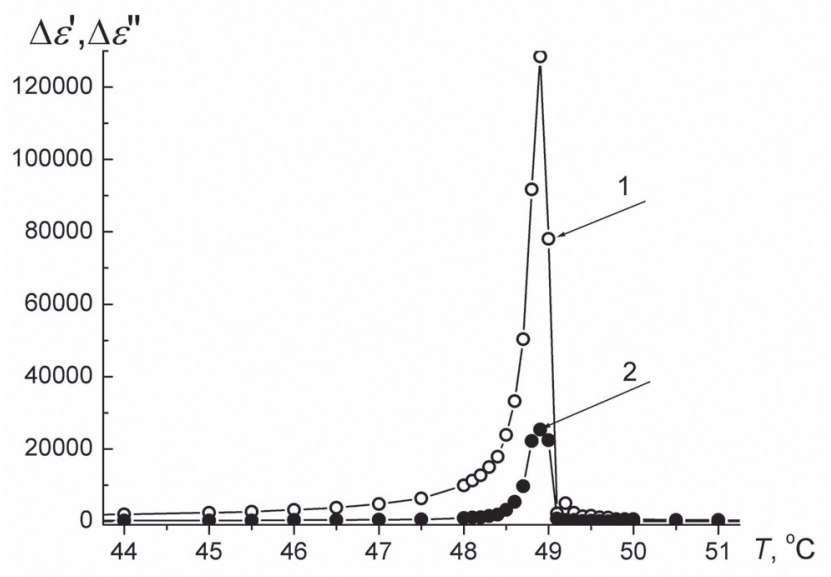

Рис. 1. Температурные зависимости разностей действительной (1) и мнимой (2) частей диэлектрической проницаемости для номинально чистого ТГС

[Fig. 1. The temperature dependences of the differences of real (1) and imaginary (2) parts of the dielectric constant for the nominally pure TGS] ях, поскольку после своего возникновения мелкая доменная структура начинает укрупняться [11-15], что ведет к уменьшению доменной компоненты, которое необходимо учитывать.

На рис. 1 для номинально чистого ТГС представлены разности температурных зависимостей $\Delta \varepsilon^{\prime}(T)=\varepsilon^{\prime}(T)_{\text {охл }}-\varepsilon^{\prime}(T)_{\text {нагр }}$, и $\left.\Delta \varepsilon^{\prime \prime} T\right)=\varepsilon^{\prime \prime}(T)_{\text {охл }}-\varepsilon^{\prime \prime}(T)_{\text {нагр }}$, измеренных при охлаждении и нагревании, и представляющие собой, с учетом сделанных оговорок, доменные компоненты $\varepsilon_{\text {дом }}^{\prime}$ и $\varepsilon_{\text {дом }}^{\prime \prime}$ или, по меньшей мере, величины очень близкие к ним.

Ненулевые значения этих величин и указывают на существенно больший вклад вынужденных осцилляций доменных стенок, возникшей при фазовом переходе неравновесной доменной структуры, в диэлектрический отклик кристалла. Как видно из рис. $2 a$, в чистом ТГС доменная компонента $\varepsilon_{\text {дом }}^{\prime}(T) \approx \Delta \varepsilon^{\prime}(T)$ зависимости $\varepsilon^{\prime}(T)_{\text {изм }}$ (аналогично $\left.\varepsilon_{\text {дом }}^{\prime \prime}(T)\right)$ наиболее велика и соизмерима с абсолютными значениями $\varepsilon^{\prime}(T)_{\text {охл }}$ (аналогично для $\left.\varepsilon_{\text {дом }}^{\prime \prime}(T)\right)$ в непосредственной близости от фазового перехода: $T_{\mathrm{C}}-T \leq 2 \mathrm{~K}$. Далее в тексте обсуждаются только зависимости, полученные для $\varepsilon^{\prime}$, поскольку для $\varepsilon^{\prime \prime}$ соответствующие зависимости качественно аналогичны.

Приведенные выше зависимости хорошо соотносятся с результатами наблюдений доменной структуры ТГС [11-15], показавшими, что после отжига в парафазе и возврата в сегнетофазу в кристалле наблюдается очень мелкая доменная структура с большим количеством доменных границ, поскольку вблизи $T_{\mathrm{C}}$ при малой величине деполяризующего поля $E_{\text {деп }} \sim P_{\mathrm{s}} / \varepsilon \varepsilon_{0}$ не требуется существенного уменьшения числа и, соответственно, энергии доменных стенок для понижения свободной энергии кристалла. Кристалл с такой мелкой доменной структурой и с большим числом доменных границ является существенно неравновесной системой, релаксирующей к равновесию, причем различие между исходным и конечным состояниями уменьшается при удалении от $T_{\mathrm{C}}[6,8]$.

Результаты измерений для ДТГС, который следует рассматривать как кристалл, в котором дефектами являются протоны, незамещенные при дейтерировании, приведены на (рис. 2б). Обращает на себя внимание более широкая по сравнению с ТГС температурная область существования доменной компоненты диэлектрической проницаемости, что, вероятно, связано с иным - более медленным характером релаксационной динамики доменных стенок в дейтерированном ТГС $[16,17]$. 

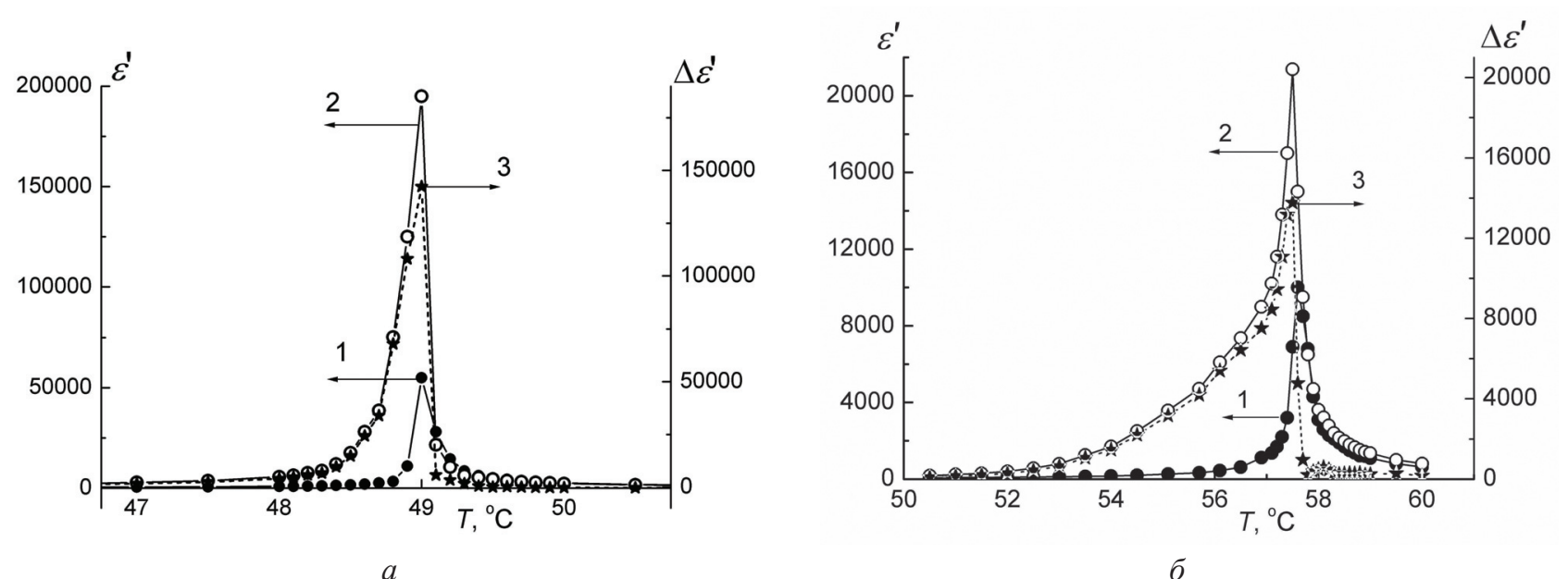

Рис. 2. Температурные зависимости: $a) \varepsilon^{\prime}$ для кристалла ТГС при нагревании - 1 и охлаждении - $2 ; 3$ - температурная зависимость $\Delta \varepsilon^{\prime}(T)=\varepsilon(T)_{\text {охл }}-\varepsilon^{\prime}(T)_{\text {нагр }}$; б) $\varepsilon^{\prime}$ для кристалла ДТГС при нагревании -1 и охлаждении $-2 ; 3-$ температурная зависимость $\Delta \varepsilon^{\prime}(T)=\varepsilon^{\prime}(T)_{\text {охл }}-\varepsilon^{\prime}(T)_{\text {нагр }}$

[Fig. 2. The dependences: a) $\varepsilon^{\prime}(T)$ for the TGS crystal at heating -1 and at cooling $-2 ; 3-$ the dependence $\Delta \varepsilon^{\prime}(T)=\varepsilon^{\prime}(T)_{\text {cool }}-\varepsilon^{\prime}(T)_{\text {heat }}$; б) $\varepsilon^{\prime}(T)$ for DTGS crystal at heating -1 and at cooling $-2 ; 3$ - the dependence

$$
\left.\Delta \varepsilon^{\prime}(T)=\varepsilon^{\prime}(T)_{\text {cool }}-\varepsilon^{\prime}(T)_{\text {heat }}\right]
$$

Дефекты разного типа и происхождения существенно изменяют статические и динамические свойства доменной структуры кристаллов ТГС [18] и других сегнетоэлектриков [16]. Введение дефектов должно сказаться и на поведении доменной составляющей измеряемых диэлектрических параметров.

На рис. 3 представлены результаты измерений для кристалла чистого ТГС, облученного дозами рентгеновского излучения: $D=48 \mathrm{kR}$ и $D=240 \mathrm{kR}$, a на рис. 4 для кристалла АТГС.

Видно, что радиационные (рис. 3) и примесные (рис. 4) дефекты уменьшают значения $\varepsilon_{\text {нагр }}, \varepsilon_{\text {охл }}$ и $\Delta \varepsilon \approx \varepsilon_{\text {дом }}$. Это влияние тем заметнее, чем выше концентрация дефектов (рис. $3 a$ и рис. 36 ), вследствие закрепления ими все большего числа доменных стенок, которые оказываются выключенными из процессов переполяризации во внешнем поле. Компонента $\varepsilon_{\text {дом }}$ в кристаллах с дефектами также локализована преимущественно вблизи $T_{\mathrm{C}}[17]$.

Абсолютная величина компоненты $\varepsilon_{\text {дом }}$ связанной с осцилляциями доменных границ в переменном электрическом поле фиксированной частоты, должна зависеть помимо величины спонтанной поляризации $[1,5]$ от плотности доменных стенок $N$ и их суммарной длины $L$ и, вероятно, увеличиваться с их ростом. Согласно термодинамической теории сегнетоэлектричества $[16,19]$, зависимость плотности доменных стенок $N$ от приведенной температуры $\Theta=\left(T_{\mathrm{C}}-T\right) / T_{\mathrm{C}}$ должна быть степенной
$N(\Theta) \sim \Theta^{-\eta}$ с показателем $\eta=0.25$. В работе [20] из зависимости $N(\Theta)$ было получено значение $\eta=0.54$, а в работе [21] из этой же зависимости для кристалла ТГС, быстро охлажденного в сегнетоэлектрическую фазу, было получено значение $\eta=1$.

Для периметра доменных границ в чистом ТГС [6] были получены степенные зависимости $L(\Theta)=a \cdot \Theta^{-\eta}$ с показателем $\eta \approx 0.5$, что согласуется с данными [20] по $N(\Theta)$.

Из закона Кюри-Вейсса $\varepsilon=C /\left(T-T_{C}\right)$ следует, что зависимость $\varepsilon(\Theta)$ может быть только степенной $\varepsilon(\Theta) \sim \Theta^{-\eta}$ с показателем $\eta=1$, что и продемонстрировано на рис. 5 для ТГС при температурах парафазы $T>T_{\mathrm{C}}$, где доменная структура отсутствует.

На рис. $6 a$ представлена зависимость $\varepsilon^{\prime}(\Theta)$ для кристалла чистого ТГС, а на рис. $6 б$ - для АТГС в сегнетоэлектрической фазе при охлаждении, когда компонента $\varepsilon_{\text {дом }}^{\prime}$ максимальна. Видно, что полученные в работе экспериментальные результаты плохо описываются степенной функцией $\varepsilon^{\prime}(\Theta)=a \cdot \Theta^{-\eta}$, тем более, с теоретическим значением показателя $\eta=0.25$, но гораздо лучше экспонентой $\varepsilon^{\prime}(\Theta)=A \cdot \exp \left(-\Theta / \Theta_{0}\right)$.

Невозможность удовлетворительно описать вблизи точки фазового перехода поведение $\varepsilon(\Theta)$ степенной функцией и наблюдающийся сильный разброс значений показателя $\eta$ для разных, исследованных в работе кристаллов группы ТГС, указывают на отсутствие полного и однозначного соответствия между статическими геометрическими параметра- 

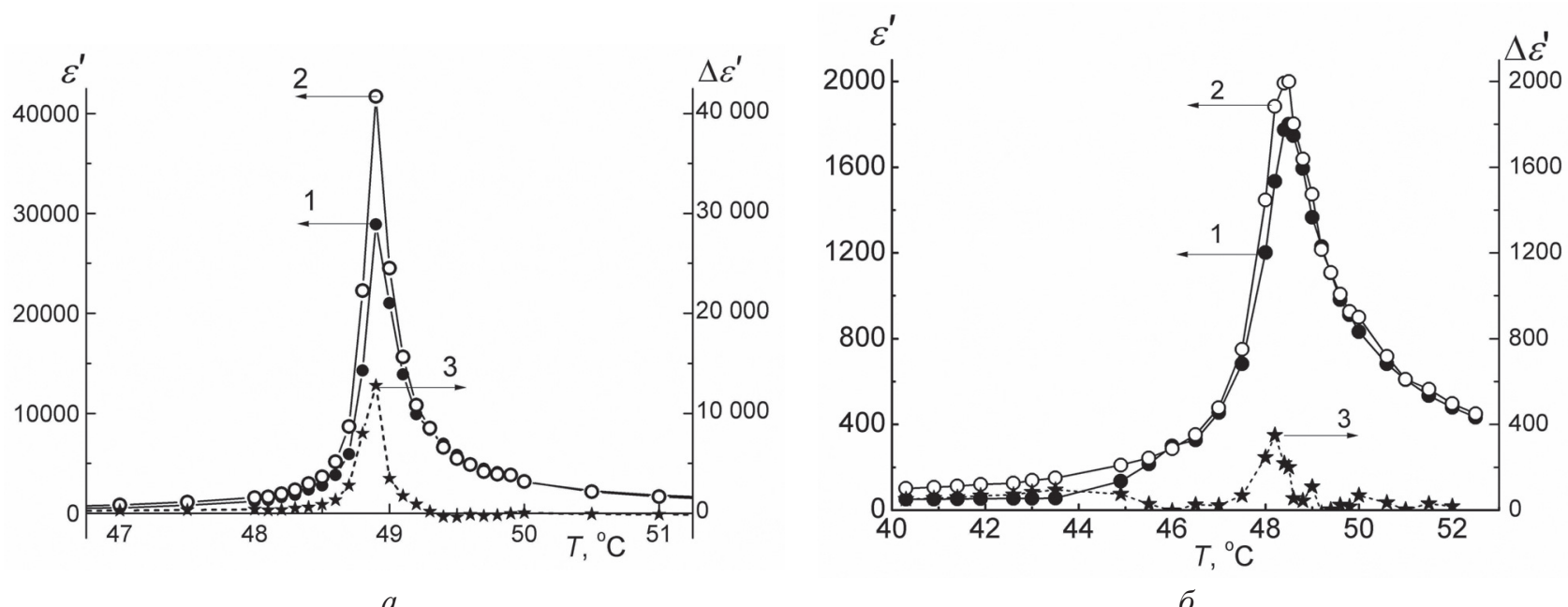

Pис. 3. Температурные зависимости $\varepsilon^{\prime}$ для кристаллов XR-TГC: при нагревании -1 и охлаждении $-2 ; 3$ - температурные зависимости $\Delta \varepsilon^{\prime}(T)=\varepsilon^{\prime}(T)_{\text {охл }}-\varepsilon^{\prime}(T)_{\text {нагр }}$. a) $D=48 \mathrm{kR}$; б) $D=240 \mathrm{kR}$

[Fig. 3. The temperature dependences of $\varepsilon^{\prime}$ for XR-TГC crystals: at heating -1 and at cooling $-2 ; 3$ - the dependence $\Delta \varepsilon^{\prime}(T)=\varepsilon^{\prime}(T)_{\text {cool }}-\varepsilon^{\prime}(T)_{\text {heat }} ;$ a) $D=48 \mathrm{kR} ;$ б) $\left.D=240 \mathrm{kR}\right]$

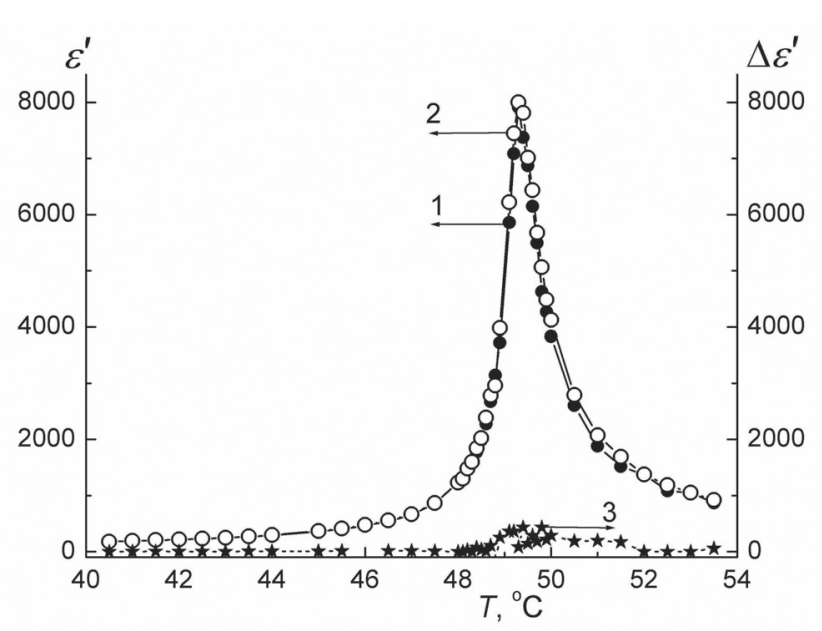

Рис. 4. Температурные зависимости $\varepsilon^{\prime}$ для кристалла АТГС: при нагревании - 1 и охлаждении - 2; 3 - температурная зависимость $\Delta \varepsilon^{\prime}(T)=\varepsilon^{\prime}(T)_{\text {охл }}-\varepsilon^{\prime}(T)_{\text {нагр }}$

[Fig. 4. The temperature dependences of $\varepsilon^{\prime}$ for ATGS: at heating -1 , at cooling $-2 ; 3$ - temperature dependence $\left.\Delta \varepsilon^{\prime}(T)=\varepsilon^{\prime}(T)_{\text {cool }}-\varepsilon^{\prime}(T)_{\text {heat }}\right]$

ми доменной структуры $(N, L)$ и измеряемыми макроскопическими характеристиками кристалла, в частности, диэлектрическими.

Основными термодинамическими закономерностями поведения диэлектрической проницаемости идеального (бездефектного) монодоменного сегнетоэлектрика с фазовым переходом второго рода в слабом переменном электрическом поле являются закон Кюри-Вейсса и «закон двойки», согласно которому углы наклона пря-

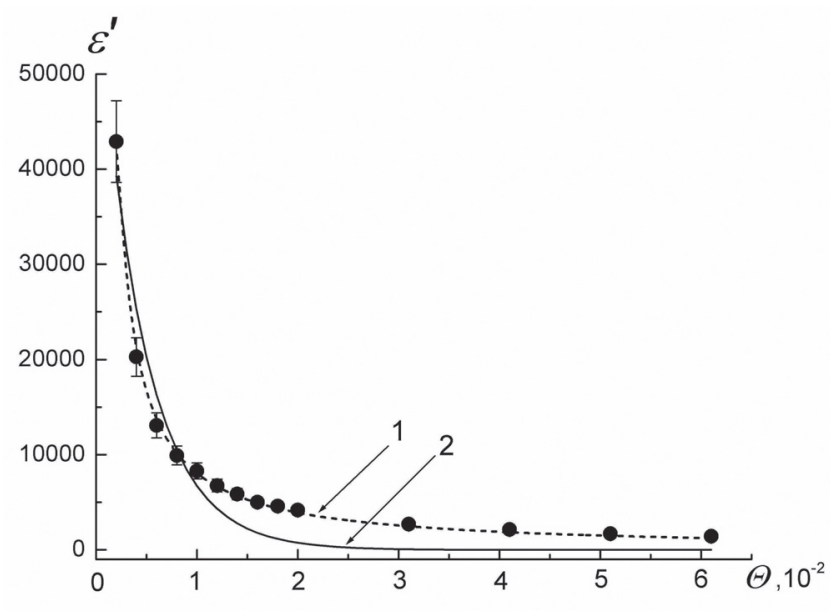

Рис. 5. Зависимость $\varepsilon^{\prime}$ от приведенной температуры при $T>T_{\text {C }}$ для кристалла ТГС при нагревании. Точки - экспериментальные значения; 1 - степенная функция $\varepsilon^{\prime}(\Theta) \sim \Theta^{-\eta}, \eta=1.03 \pm 0.01 ; 2-$ экспонента

[Fig. 5. The dependence of $\varepsilon^{\prime}$ on the reduced temperature $\Theta$ at $T>T_{\mathrm{C}}$ for TGS crystal at heating. Points - experimental values; 1 - power function $\varepsilon^{\prime}(\Theta) \sim \Theta^{-\eta} ; \eta=1.03 \pm 0.01 ; 2-$ an exponential function]

мых $1 / \varepsilon^{\prime}(T)$ по обе стороны от $T_{\mathrm{C}}$ отличаются вдвое. В реальном эксперименте возможны небольшие отклонения от «закона двойки», допускающие значения $2.7 \div 2.8$ и вызванные нарушением условий изотермичности фазового перехода и влиянием механического зажатия кристалла [18, 22].

Для номинально чистого ТГС (рис. 7a) и ДТГС закон Кюри-Вейсса (линейность зависимости $\left.\varepsilon^{-1}(T)\right)$ хорошо выполняется в обеих фазах. Закон «двой- 

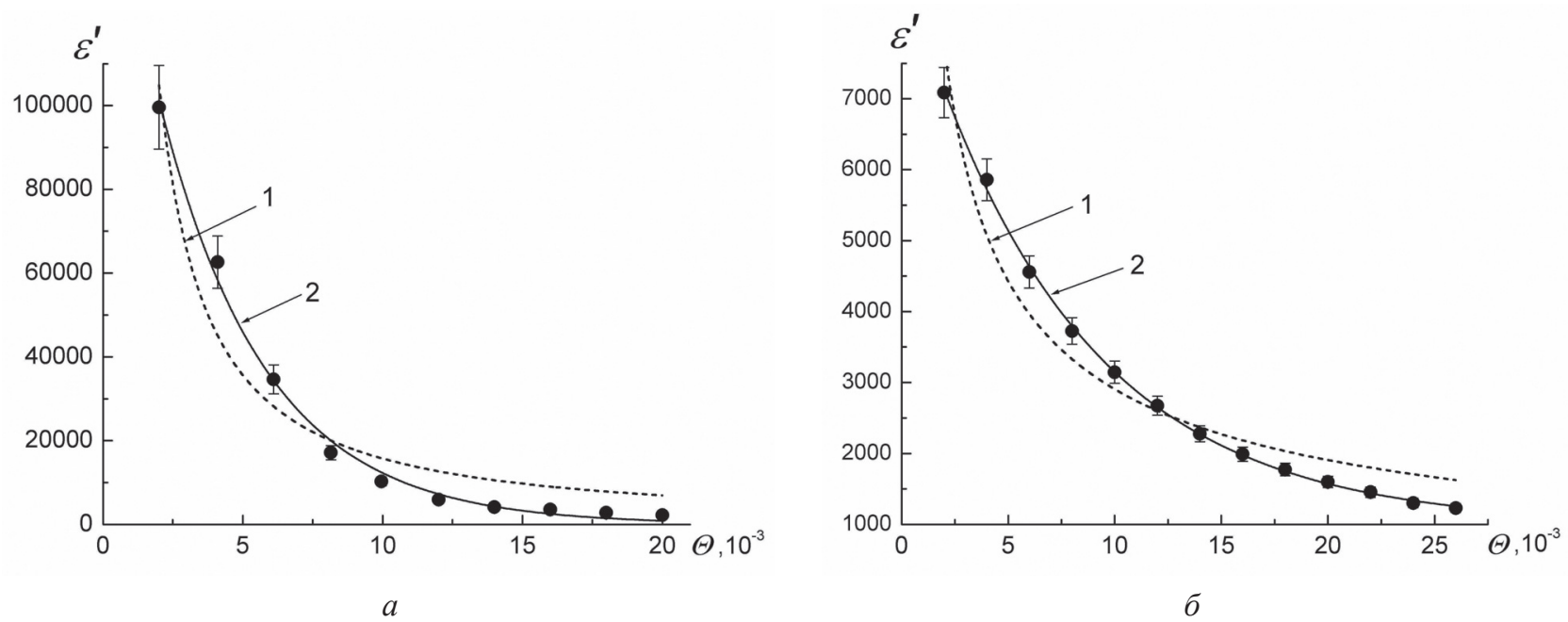

Рис. 6. Зависимость $\varepsilon^{\prime}$ от приведенной температуры для кристалла ТГС - a) и АТГС - б) при охлаждении. Точки - экспериментальные зависимости; линии: 1 - степенная функция, 2 -экспонента

[Fig. 6. The dependences of $\varepsilon^{\prime}$ on the reduced temperature $\Theta$ during cooling for: $a$ ) TGS, б) ATGS Points - experimental data; lines: 1 - a power function, 2 - an exponential function]

ки» удовлетворительно выполняется в режиме нагревания, когда компонента $\varepsilon_{\text {дом }}$ мала, и нарушается в режиме охлаждения при появлении значительной $\varepsilon_{\text {дом }}$. При этом значение константы Кюри в сегнетоэлектрической фазе $C^{\mathrm{F}}$ существенно возрастает (рис. 7 а, прямая 2). Нарушение этого закона наблюдается также в слабодефектных кристаллах ТГСФ и XR-TГC с малыми дозами облучения. В высокодефектных кристаллах - АТГС и XR-ТГС с большой дозой облучения (рис. 7б) - хорошо выполняются оба закона. Таким образом, термодинамические законы, справедливые для идеального мо-

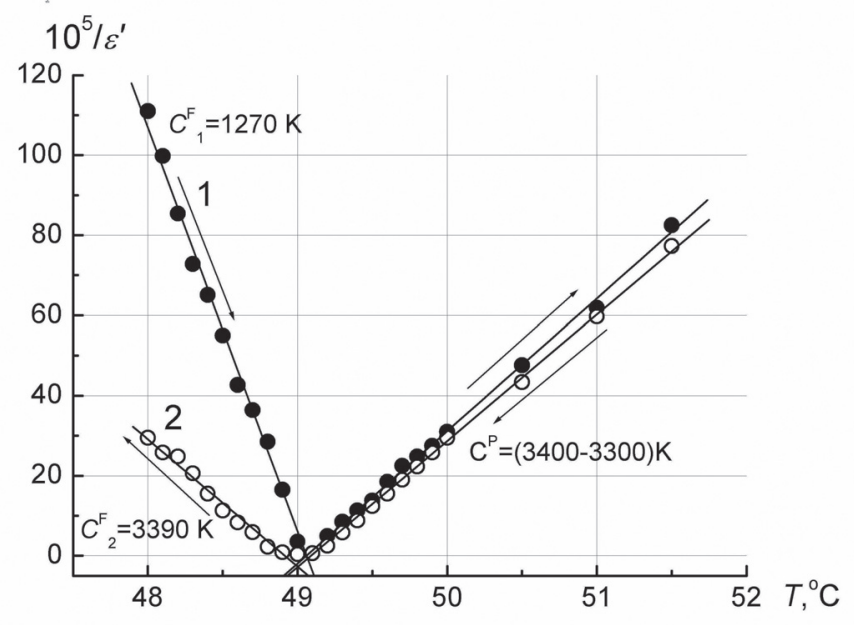

$a$ нодоменного сегнетоэлектрика $[16,19]$, нарушаются в кристалле с подвижной во внешнем поле доменной структурой, но выполняются в кристалле со структурными дефектами, закрепляющими доменные стенки.

Рассмотренное выше влияние кратковременного отжига в парафазе, приводящего после возвращения в сегнетоэлектрическую фазу к резкому увеличению доменной компоненты диэлектрических параметров, может варьироваться при изменении скорости прохождения фазового перехода и при увеличении числа термических циклов. На рис. $8 a$ при-

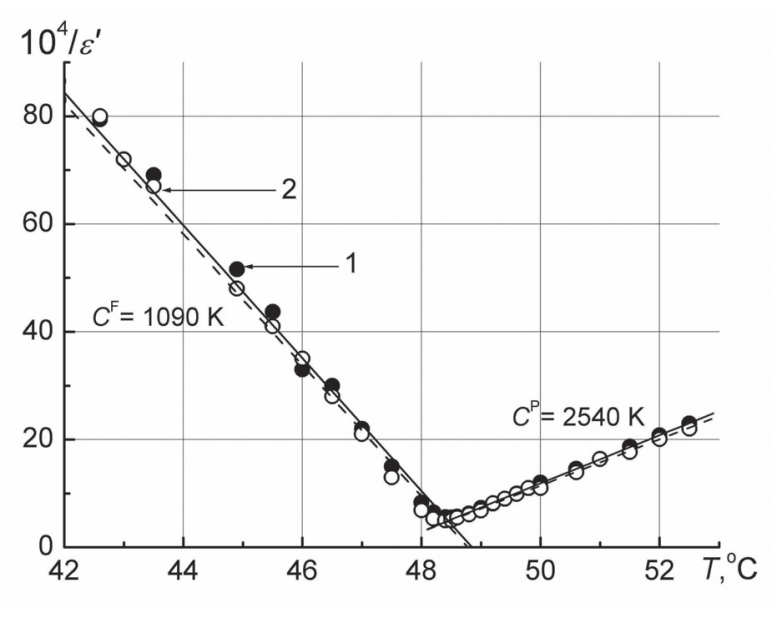

6

Рис. 7. Зависимость $\left.1 / \varepsilon^{\prime}(T): a\right)$ TГC, б) ХR-TГC ( $\left.D=240 \mathrm{kR}\right) ; 1$ - нагревание, 2 - охлаждение. Точки - экспериментальные данные. Около прямых представлены константы Кюри в парафазе $C^{\mathrm{p}}$ и сегнетофазе $C^{\mathrm{F}}$

[Fig. 7. The dependences of $\left.1 / \varepsilon^{\prime}(T): a\right)$ TGS, б) XR-TGS $(D=240 \mathrm{kR})$. Points - the experimental data at heating - 1 and cooling -2 . $C^{\mathrm{P}}$ and $C^{\mathrm{F}}$ are the Curie constants respectively in paraelectric phase and in ferroelectric phase] 

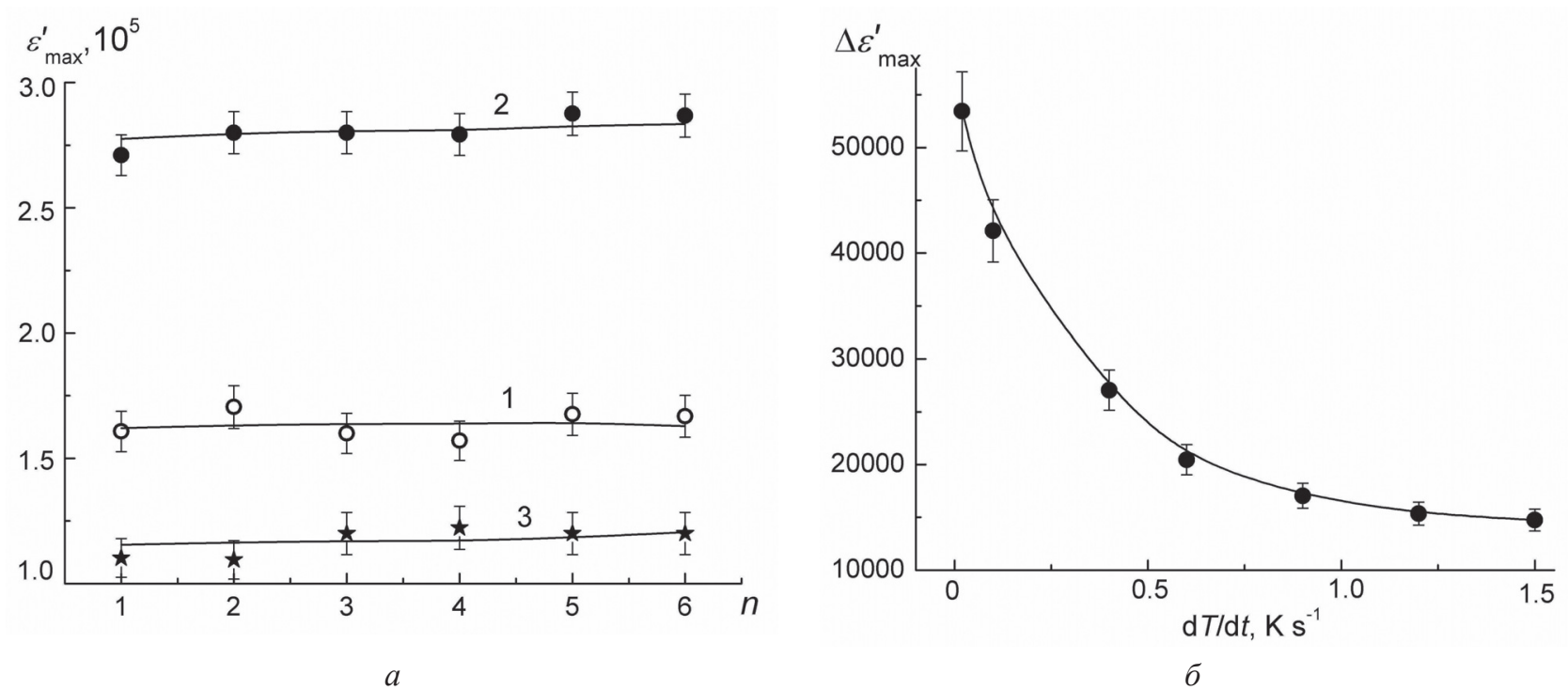

Рис. 8. Зависимости: a) $\varepsilon_{\text {max }}^{\prime}$ чистого ТГС от числа переходов $n$ через $T_{\mathrm{C}}$ со скоростью $\sim 0.5 \mathrm{~K} \cdot \mathrm{s}^{-1}: 1-$ в параэлектрическую фазу, 2 - в сегнетоэлектрическую фазу, 3 - разность $\Delta \varepsilon^{\prime}{ }_{\text {max }}$ кривых 2 и 1; б) $\Delta \varepsilon^{\prime}{ }_{\text {max }}$ от скорости перехода через $T_{\mathrm{C}}$ для чистого ТГС

[Fig. 8. The dependences for pure TGS crystal: $a$ ) of $\varepsilon^{\prime}{ }_{\text {max }}$ on the number of passages $\mathrm{n}$ through $T_{\mathrm{C}}$ at a rate $\mathrm{d} T / \mathrm{d} t \approx 0.5 \mathrm{~K} \cdot \mathrm{s}^{-1}$ : 1 - into the paraelectric phase, 2 - into the ferroelectric phase, $3-\Delta \varepsilon_{\max }(n)$ the difference between curves 2 and 1 ; $\sigma$ ) of $\Delta \varepsilon^{\prime}{ }_{\text {max }}$ on the rate of transition through $T_{\mathrm{C}}$ ]

ведены зависимости $\varepsilon_{\max }^{\prime}$ и $\Delta \varepsilon_{\max }^{\prime}=\left(\varepsilon_{\max }^{\prime}\right)_{\text {охл }}-\left(\varepsilon_{\max }^{\prime}\right)_{\text {нагр }}$ для чистого кристалла ТГС от числа термоциклирований $n$. Видно, что этот фактор практически не влияет на величину доменной составляющей, по крайней мере, в точке Кюри. Напротив, увеличение скорости прохождения фазового перехода, как следует из рис. 8б, приводит к заметному понижению этой величины.

\section{2. СЕГНЕТОВА СОЛЬ}

На рис. 9 представлена зависимость $\Delta \varepsilon^{\prime}(T)$ для кристалла сегнетовой соли (здесь

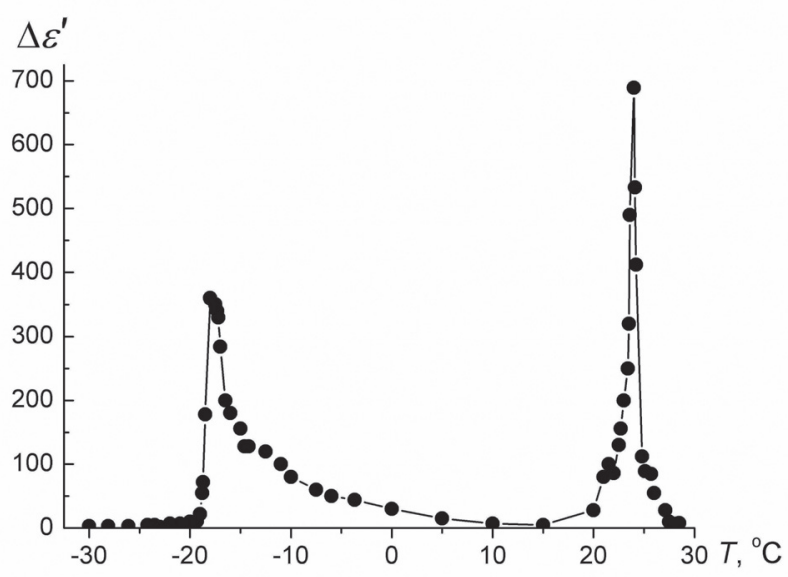

Рис. 9. Температурная зависимость $\Delta \varepsilon^{\prime}$ для кристалла сегнетовой соли

[Fig. 9. A temperature dependence of $\Delta \varepsilon^{\prime}$ for Rochelle salt crystal]
$\Delta \varepsilon^{\prime}(T)==\varepsilon^{\prime}(T)_{\text {нагр }}-\varepsilon^{\prime}(T)_{\text {охл }}$ в области $T_{\mathrm{C1}}$ и $\Delta \varepsilon^{\prime}(T)=\varepsilon^{\prime}(T)_{\text {охл }}-\varepsilon^{\prime}(T)_{\text {нагр }}$ в окрестности $\left.T_{\mathrm{C} 2}\right)$. Видно, что величина доменного вклада $\varepsilon_{\text {дом }}^{\prime} \approx \Delta \varepsilon^{\prime}$ вблизи $T_{\mathrm{C} 2}$ по абсолютной величине заметно выше, чем вблизи $T_{\mathrm{Cl}}$, и так же, как в кристаллах ТГС, существенна только в узком температурном интервале, в то время как вблизи $T_{\mathrm{Cl}}$ меньший по величине доменный вклад в $\varepsilon$ проявляется в заметно более широком температурном интервале.

Большая ширина температурной области существования доменной компоненты вблизи нижней точки Кюри связана, вероятно, с тем, что в этом температурном интервале имеет место иная, более замедленная, нежели вблизи верхней точки Кюри, динамика доменных границ [23]. Она характеризуется более высокими значениями времен релаксации доменных процессов, и энергий активации термостимулированного движения доменных границ [24].

Закон Кюри-Вейсса (рис. 10) хорошо выполняется вблизи обеих точек Кюри, как в параэлектрических, так и в сегнетоэлектрической фазе. «Закон двойки» в его классическом виде выполняется вблизи $T_{\mathrm{C} 2}$ (рис. 10б) и не выполняется вблизи $T_{\mathrm{C} 1}$ (рис. $10 a$ ). Столь же сильного, как в кристаллах ТГС, влияния доменной составляющей $\varepsilon$ на выполнимость указанных законов не наблюдается. 


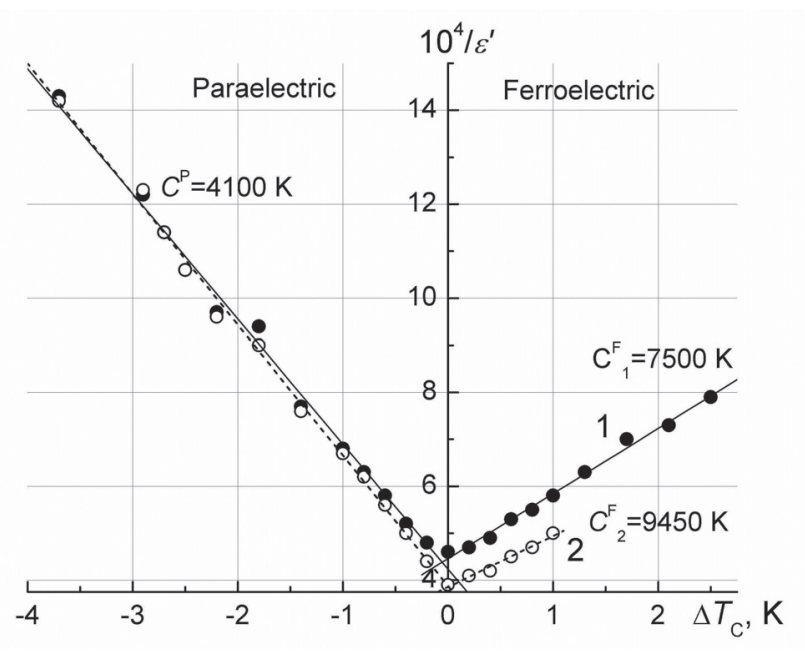

$a$

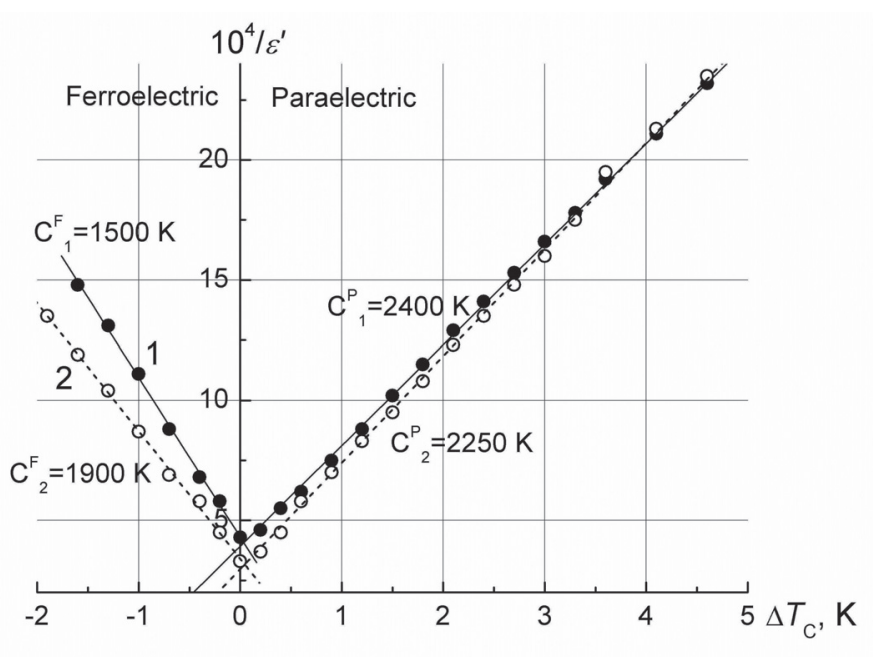

$\sigma$

Рис. 10. Температурные зависимости обратной диэлектрической проницаемости кристалла сегнетовой соли: a) вблизи $T_{\mathrm{C} 1}$ при охлаждении - 1 и нагревании $\left.-2 ; \sigma\right)$ вблизи $T_{\mathrm{C} 2}$ при нагревании -1 и охлаждении -2

[Fig. 10. A temperature dependence of $1 / \varepsilon^{\prime}$ for Rochelle salt crystal: $a$ ) near $T_{\mathrm{Cl}}$ during cooling -1 and heating $-2 ; \sigma$ ) near $T_{\mathrm{C} 2}$ during heating -1 and cooling -2 ]

\section{ВЫВОДЫ}

1. Для кристаллов ТГС - чистого, с примесными и радиационными дефектами, а также для кристалла сегнетовой соли найдены температурные зависимости доменных компонент действительной и мнимой частей диэлектрической проницаемости, измеренных в слабом переменном электрическом поле. Эти зависимости получены как разности температурных зависимостей $\varepsilon^{\prime}$ и $\varepsilon^{\prime \prime}$, измеренных: a) при охлаждении в сегнетоэлектрическую фазу образца, выдержанного в параэлектрической фазе; б) при нагревании образца, выдержанного в сегнетоэлектрической фазе вдали от $T_{\mathrm{C}}$.

2. Возможность выделения доменной составляющей определена существенным различием доменных конфигураций (число и длина доменных границ, их подвижность в переменном электрическом поле) состаренного и омоложенного образца.

3. В чистом ТГС доменные компоненты $\varepsilon_{\text {дом }}^{\prime}(T)$ и $\varepsilon_{\text {дом }}^{\prime \prime}(T)$ наиболее велики и соизмеримы с абсолютными значениями $\varepsilon^{\prime}(T)_{\text {охл }}$ и $\varepsilon^{\prime \prime}(T)_{\text {охл }}$ в непосредственной близости от фазового перехода: $T_{\mathrm{C}}-T \leq 2 \mathrm{~K}$.

4. Примесные и радиационные дефекты подавляют доменный вклад в измеряемые диэлектрические параметры, вследствие чего, в таких кристаллах лучше выполняются классические законы термодинамической теории: закон Кюри-Вейсса и «закон двойки».

5. Для исследованных кристаллов группы ТГС отсутствует количественное соответствие между температурным поведением статических геометрических параметров доменной структуры (плотность $N$ и полная длина $L$ доменных границ) и измеряемых макроскопических характеристик, в частности, диэлектрических, вблизи температуры фазового перехода.

6. Число термических циклов нагревания - охлаждения с переходом через точку Кюри практически не влияет на величину доменной составляющей $\varepsilon_{\text {дом }}$ (по крайней мере, в точке Кюри) в кристаллах ТГС. Увеличение скорости прохождения фазового перехода, приводит к заметному понижению этой величины.

7. В кристалле сегнетовой соли величина доменного вклада $\varepsilon_{\text {дом }}$ вблизи верхней $T_{\mathrm{C}}$ по абсолютной величине заметно выше, чем вблизи нижней $T_{\mathrm{C}}$, и так же, как в кристаллах ТГС, существенна только в узком температурном интервале. Вблизи нижней $T_{\mathrm{C}}$ меньший по величине доменный вклад в $\varepsilon$ занимает более широкий температурный интервал, поскольку при этих температурах динамика доменных границ является более замедленной.

\section{СПИСОК ЛИТЕРАТУРЫ}

1. Сидоркин А. С. Доменная структура в сегнетоэлектриках и родственньх материалах. М.: Наука, Физматлит, 2000, 240 с.

2. Tagantsev A. K., Cross L. E., J. Fousek J. Domains in Ferroic Crystals and Thin Films Springer, New York, 2010, 830 p.

3. Kamysheva L. N., Drozhdin S. N. // Ferroelectrics, 1987, vol. 71, pp. 281-296.

4. Камышева Л. Н., Дрождин С. Н., Голицына О. М. // ФTT, 1998, т. 40, № 1, с. 116-117 
5. Лотонов А. М., Новик В. К., Гаврилова Н. Д. // ФTT, 2006, т. 48, с. 969-972.

6. Дрождин С. Н., Голицына О. М. // ФТТ, 2012, т. 54, c. $853-858$.

7. Голицына О. М., Дрождин С. Н., В. О. Чулакова В. О. // Известия РАН. Серия Физическая, 2016, т. 80, № 9, с. 1214-1217.

8. Голицына О. М., Гречкина М. В., Дрождин С. Н., Чулакова В. О. // Конденсированные среды и межфазные гранииьы, 2016, т. 18, № 4, с. 494-504.

9. Kamysheva L. N., Drozhdin S. N., Serdyuk O. M. // Phys. Stat. Sol. (a), 1986, vol. 97, K29-K34.

10. Камышева Л. Н., Дрождин С. Н., Голицына О. М. // Вестник ВГТУ, Серия «Физическое материаловедение»,1996, т. 1, № 1, с. 96-107.

11. Tomita N., Orihara H., and Ishibashi Y. // J. of the Phys. Society of Jap., 1989, vol. 58, pp. 1190-1196.

12. Likodimos V., Labardi V., and Allegrini M. // Phys. Review B, 2000, vol. 61, № 21, pp. 14440-14447.

13. Golitsyna O. M., Drozhdin S. N., Chulakova V. O., Grechkina M. V. // Ferroelectrics, 2017, vol. 506, 127-135.

14. Голицына О. М., Дрождин С. Н., Коробова А. Д., Чулакова В. О. // Конденсированные среды и межфазные гранииы, 2017, т. 19, № 1, с. 42-50.

15. Nakatani N. // Japan. J. of Appl. Phys., 1985, vol. 24, № 7, pp. L528-L530.

16. Лайнс М., Гласс А. Сегнетоэлектрики и родственные материалы. М.: Мир, 1981, 736 с.

17. Дрождин С. Н., Куянцев М. А. // ФTT, 1998, т. 40, № 8, c. 1542-1545.

18. Цедрик М. С. Физические свойства кристаллов семейства триглицинсульфата. Минск, Наука и техника, 1986, 216 с.

19. Струков Б. А., Леванюк А. П. Физические основы сегнетоэлектрических явлений в кристаллах. М.: Наука, Физматлит, 1995, 216 с.

20. Luo E. Z., Xie Z., Xu J. B., Wilson I. H. // Physical Review B., 2000, vol. 61, № 1, pp. 203-206.

21. Nakatani N. // Japan. J. of Appl. Phys., 1985, vol. 24, № 7, pp. L528-L530.

22. Иона Ф., Ширане Д. Сегнетоэлектрические кристалльь. М.: Мир, 1965, 554 с.

23. Шильников А. В. // Известия. АН СССР. Серия физическая, 1987, т. 51, с. 1726-1729.

24. Голицына О. М., Дрождин С. Н., Никишина А. И., ФТТ, 2007 , т. 49, вып. 10, с. 1862-1865.

\title{
DOMAIN CONTRIBUTION TO THE DIELECTRIC PROPERTIES OF THE CRYSTALS OF TRIGLYCINE SULPHATE GROUP AND ROCHELLE SALT
}

\author{
(C) 2017 O. M. Golitsyna, S. N. Drozhdin, V. O. Chulakova \\ Voronezh State University, 1 University sq., 394018 Voronezh, Russia \\ e-mail:drozhdin@phys.vsu.ru
}

Received 31.03.2017

\begin{abstract}
The dielectric properties of the crystals of nominally pure triglycine sulphate (TGS), deuterated TGS, TGS doped with L, $\alpha$ - alanine, TGS with impurity of phosphorus $\mathrm{P}^{5+}$ ions, TGS subjected to X-ray radiation as well as of Rochelle salt crystal were investigated. Temperature dependences of domain components of the real and imaginary parts of the dielectric permittivity measured in a weak $a c$ electric field were obtained. The existence of a moveable domain structure in defect-free TGS crystals does not lead to a deviation from the thermodynamic Curie - Weiss law in the ferroelectric phase, but causes a violation of the "law of two". In TGS crystals with impurity or radiation defects, pinning the domain structure, the both laws work well. For the studied crystals of the TGS group near the phase transition, there is no quantitative correlation between temperature behavior of static geometric parameters of the domain structure (density $N$ and total length $L$ of the domain boundaries), and the measured dielectric characteristics. The number of thermal cycles of heating and cooling with a transition through the Curie point practically does not affect the value of the domain component of the dielectric constant in TGS crystals, while an increase of the rate of passage of the phase transition leads to its significant decrease. In Rochelle salt crystals the domain part of the dielectric permittivity near the lower phase transition is considerably less than near the upper one, but it occupies a wider temperature range.
\end{abstract}

Keywords: triglycine sulphate, Rochelle salt, domain structure, oscillations of domain boundaries, dielectric properties, defects, Curie - Weiss law, "the law of two". 


\section{REFERENCES}

1. Sidorkin A. S. Domain Structure in Ferroelectrics and Related Materials. United Kingdom, Cambridge International Science Publising, 2006, 234 p.

2. Tagantsev A. K., Cross L. E., J. Fousek J. Domains in Ferroic Crystals and Thin Films Springer, NewYork, $2010,830 \mathrm{p}$.

3. Kamysheva L. N., Drozhdin S. N. Ferroelectrics, 1987, vol. 71, pp. 281-296.

4. Golitsyna O. M., Kamysheva L. N., Drozhdin S. N. Physics of the Solid State, 1998, vol. 40, no. 1, pp. 103-104. DOI: 10.1134/1.1130245

5. Lotonov A. M., Novik V. K., Gavrilova N. D. Physics of the Solid State, 2006, vol. 48, no. 6, pp. 1030-1033. DOI: $10.1134 / \mathrm{S} 1063783406060047$

6. Drozhdin S. N., Golitsyna O. M. Physics of the Solid State, 2012, vol. 54, no. 5, pp 905-910. DOI: 10.1134/ S1063783412050071

7. Golitsyna O. M., Drozhdin S. N., CHulakova V. O. Bulletin of the Russian Academy of Sciences: Physics, 2016, vol. 80, no. 9, pp. 1111-1114. DOI: 10.7868/ S0367676516090131

8. Golitsyna O. M., Grechkina M. V., Drozhdin S. N., Chulakova V. O. Condensed Matter and Interphases, 2016, vol. 18, no. 4, pp. 494 -504. Available at: http://www.kcmf. vsu.ru/resources/t_18_4_2016_006.pdf(in Russian)

9. Kamysheva L. N., Drozhdin S. N., Serdyuk O. M. Phys. Stat. Sol.(a), 1986, vol. 97, K29-K34.

10. Kamysheva L. N., Drozhdin S. N., Golitsyna O. M. Vestnik VSTU, Series “Physical Materials Science”,1996, vol. 1, no. 1, pp. 96-107.

11. Tomita N., Orihara H., and Ishibashi Y. J. of the Phys. Society of Jap., 1989, vol. 58, p. 1190-1196.

12. Likodimos V., Labardi V., and Allegrini M. Phys. Review B, 2000, vol. 61, no. 21, pp. 14440-14447. DOI: 0163-1829/2000/61 21!/14440 8!/\$15.00
13. Golitsyna O. M., Drozhdin S. N., Chulakova V. O., Grechkina M. V. Ferroelectrics, 2017, vol. 506, pp. 127-135. DOI: $10.1080 / 00150193.2017 .1282286$

14. Golitsyna O. M., Drozhdin S. N., Korobova A. D., Chulakova V.O. Condensed Matter and Interphases, 2017, vol. 19, no. 1, pp. 42-50. Available at: http://www.kcmf. vsu.ru/resources/t_19_1_2017_005.pdf(in Russian)

15. Nakatani N. Japan. J. of Appl. Phys., 1985, vol. 24, no. 7, pp. L528-L530.

16. Lines M. E. and Glass A. M. Principle and Applications of Ferroelectrics and Related Materials. New York, Clarendon, 1982, 680 p.

17. Drozhdin S. N., Kuyancev M. A. Physics of the Solid State, 1998, vol. 40, no. 8, pp. 1398-1401. DOI: 10.1134/1.1130567

18. Tsedrik M. S. Physical Properties of Crystals of the Triglycine Sulfate Family. Minsk, Science and Technology Publ., 1986, 216 p. (in Russian)

19. Strukov B. A., Levanyuk A.P. Physical Fundamentals of Ferroelectric Phenomena in Crystals. Moscow: Nauka, Fizmatlit Publ., 1995, 216 p. (in Russian)

20. Luo E. Z., Xie Z., Xu J. B., Wilson I. H. Physical Review B., 2000, vol. 61, no. 1, pp. 203-206. DOI: https:// doi.org/10.1103/PhysRevB.61.203

21. Nakatani N. Japan. J. of Appl. Phys., 1985, vol. 24, no. 7, pp. L528-L530.

22. Jona F., Shirane G. Ferroelectric Crystals. New York, Pergamon press, 1962, 554 p.

23. SHil'nikov A. V. Bulletin of the Russian Academy of Sciences: Physics, 1987, vol. 51, pp. 1726-1729. (in Russian)

24. Golitsyna O. M., Drozhdin S. N., Nikishina A. I. Physics of the Solid State, 2007, vol. 49, no. 10, pp. 19531956. DOI: 10.1134/S106378340710023X
Голицына Ольга Михайловна - к. ф.-м. н., доцент, Воронежский государственный университет; тел.: +7(473) 2208625, e-mail: golitsynaom@yandex.ru

Дрождин Сергей Николаевич - д. ф.-м. н., профессор, заведующий кафедрой, Воронежский государственный университет; тел.:+7(919) 1824460, e-mail: drozhdin@ phys.vsu.ru

Чулакова Валерия Олеговна-аспирант, Воронежский государственный университет; тел.: +7(473) 2208625 , e-mail: chulakovavo@mail.ru

Гриднев Александр Евгеньевич - ассистент, Воронежский государственный университет; тел.: +7(473) 2208625, e-mail: gridnev@phys.vsu.ru
Golitsyna Olga M. - Cand. Sci. (Phys.-Math.), Assistant Professor, Voronezh State University, ph.: +7(473) 2208625, e-mail: golitsynaom@yandex.ru

Drozhdin Sergey N. - Dr. Sci. (Phys.-Math.), Professor, Head of Department, Voronezh State University, ph.: +7(919) 1824460, e-mail: drozhdin@phys.vsu.ru

Chulakova Valeriya O. - postgraduate student, Voronezh State University, ph.: +7(473) 2208625, e-mail: chulakovavo@mail.ru

Gridnev Aleksandr E. - Assistent, Voronezh State University; ph.: +7(473) 2208625, e-mail: gridnev@phys. vsu.ru 